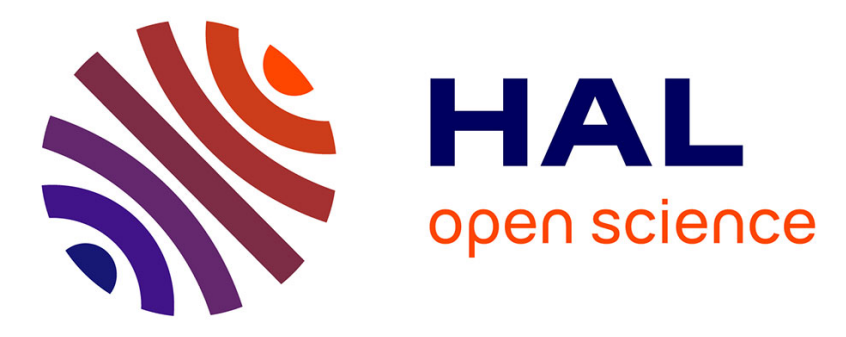

\title{
Advanced hybrid tracking through neural network regression
}

M'Hamed Frad, Hichem Maaref, Abdellatif Matibaa, Samir Otmane

\section{To cite this version:}

M'Hamed Frad, Hichem Maaref, Abdellatif Matibaa, Samir Otmane. Advanced hybrid tracking through neural network regression. 2014 Information and Communication Technologies Innovation and Application (ICTIA 2014), Mar 2014, Sousse, Tunisia. 10.1109/ICTIA.2014.7883766 . hal01777621

\section{HAL Id: hal-01777621 \\ https://hal.science/hal-01777621}

Submitted on 18 Jul 2018

HAL is a multi-disciplinary open access archive for the deposit and dissemination of scientific research documents, whether they are published or not. The documents may come from teaching and research institutions in France or abroad, or from public or private research centers.
L'archive ouverte pluridisciplinaire HAL, est destinée au dépôt et à la diffusion de documents scientifiques de niveau recherche, publiés ou non, émanant des établissements d'enseignement et de recherche français ou étrangers, des laboratoires publics ou privés. 


\title{
Advanced hybrid tracking through neural network regression
}

\author{
${ }^{1,2}$ M'hamed Frad, ${ }^{1}$ Hichem Maaref, ${ }^{2}$ Abdellatif Matibaa, ${ }^{1}$ Samir Otmane, \\ ${ }^{1}$ IBISC Laboratory, Evry Val d'Essonne University, France \\ ${ }^{2}$ E $\mu$ E Laboratory, Monastir University, Tunisia \\ \{firstname\}.\{name\}@ibisc.fr, abdellatif.mtibaa@enim.rnu.tn
}

\begin{abstract}
- we present an optical-haptic tracking system suitable for augmented and virtual reality applications. The paper addresses tow issues of such system. The first one concerns the calibration method that can be used to calibrate the force feedback device: SPIDAR. The second contribution is about the development of a hybrid tracking system. The proposed hybridization aims to provide both accurate and interrupted position data.
\end{abstract}

Keywords-SPIDAR, Calibration, Regression, Neural Network hybrid tracking

\section{INTRODUCTION}

The SPIDAR [1], for SPace Interaction Device for Augmented Reality, is a kind of motor string-based haptic display system. The system allows the user to directly manipulate virtual object in the virtual environment. The proposed system is called SPIDAR- 8 , which has 8 couples of DC motor/rotary encoder distributed on each vertex of a cubic structure. Position of the user can be measured by the length of the strings. The length of a string is known by reading the values from the rotary encoder. Nevertheless, the SPIDAR tracking is highly distorted and inaccurate.

The suitable device used in virtual reality and augmented reality systems, as well as ours, is the optical tracking system. However, it has a major drawback: tracking-loss. The combination of the SPIDAR and the Optical tracking system allows to overcome drawbacks of both tracking technologies and to make 3D interaction to be more vivid and continuous. In this research paper in due hand, we underline first previous works on tracking system. Then, we describe the method used for registration of the tracking system to report data position. Thereafter, we develop a calibration and registration algorithm for providing special accuracy of both tracking system component. Lastly, we evaluate the performances of the proposed hybridization method.

\section{RELATED WORKS}

The following overview of related activities focuses mainly on the calibration methods for position sensor systems.

Further references are discussed with regard to similar to our approach. Early works have focused on electromagnetically based tracking systems that are sensitive to the ambient electromagnetic environment.

Bryson [2], in a technical report, has discussed methods for the measurement and characterization of the static distortion in $3 \mathrm{D}$ tracking system. The measurement and calibration methods were applied to the Polhemus electromagnetic tracking system Kindratenko and Sherman [3] have performed a calibration method based on neural network for electromagnetic tracking systems. They obtained quite good results compared to other calibration methods like linear interpolation.

Barratt et al [4] have proposed an electromagnetic 3D ultrasound system for vascular imaging application. They use a robust registration method developed by Fitzgibbon [5].

Livingston [6] used a mapping gird to calibrate the electromagnetic tracking systems. He restricted the workspace to improve its accuracy. He succeeded to reduce error position to $79 \%$. Birkfellner et al [7] developed a hybrid tracking system based on both electromagnetic and optical used in surgery. The purpose of such system is to ensure the tracking continuity as well as to overcome the limits of both systems.

\section{SPIDAR CHARACTERIZATION}

\section{The measurement process}

For a properly SPIDAR characterization, the three dimensional position must not depend on technical constraints. Therefore, we use a virtual grid composed of a huge number of small cubes, implying that collected SPIDAR data covers the whole SPIDAR workspace.

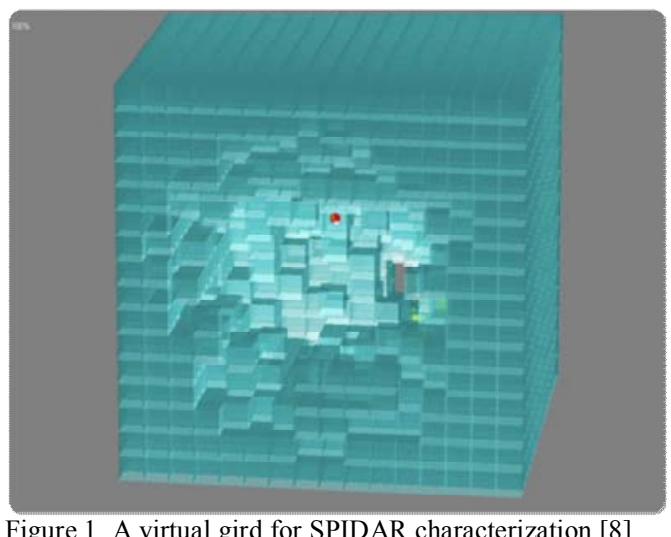

Figure 1. A virtual gird for SPIDAR characterization [8] 
To perform the suggested characterization, two infrared cameras need to be rigidly mounted on the platform used, for instance the big SPIDAR. We call this optical tracking system "ART Tracking".

Data subsequently needs to be collected while moving the effector in all possible orientations. Whenever the effector collides with a cube, we record the positions given by SPIDAR, the positions given by ART tracking system and also the encoder values. The above measurements were performed in a workspace limited to $1 \mathrm{~m}^{3}$ to obtain good characterization.

\section{Three-dimensional structure of the SPIDAR distortion}

Distortions can be viewed in several ways. The clearest way of representing of this distortion is with a position error, defined as the returned position by the tracker minus the actual position. The three dimensional graphic below (Fig 3) indicates this distortion. The distribution of absolute error seems to be onion skin distribution in the sense that error is distributed over many spherical planes. The magnitude of absolute error grows as the effector is going far from the center of the measurement volume. In fact, the magnitude of error is longer than $30 \mathrm{~mm}$ in outside planes.

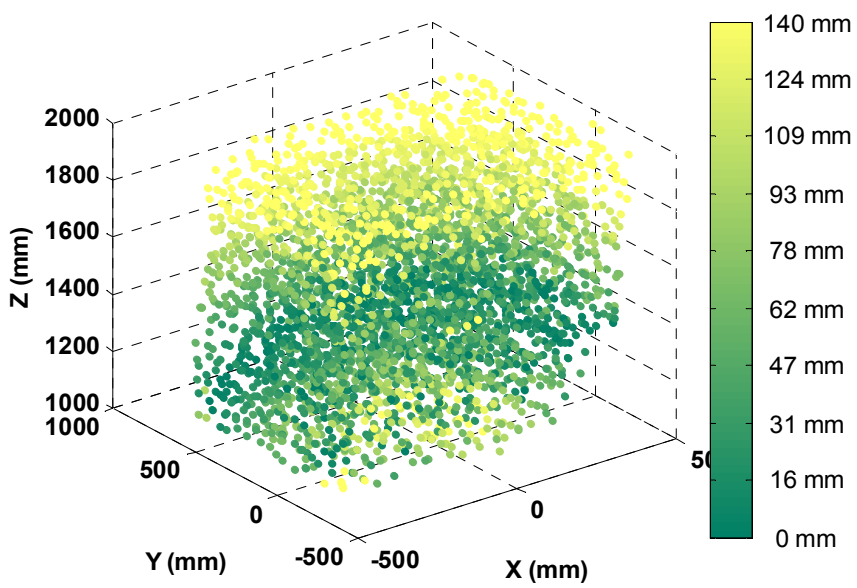

Fig. 2. Absolute Error distribution in the SPIDAR workspace (Dark green is the best).

The characterization of SPIDAR leads us to different problems. On one hand, SPIDAR has several defects that distort position measurements. On the other hand, SPIDAR interface is a black box. So, we have no idea on the mathematical model used to compute effector position.

\section{NEURAL NETWORK CALIBRATION METHOD}

This paper presents a regression-based approach for solving the SPIDAR's calibration problem. In statistics, regression is composed of techniques for modeling the relationship between a response variable and one or more explanatory variables [8]. The goal is to find a useful function $f(x)$ for the regression function $f(x)$ that estimates effector position in terms of value of observed responses. The following discussion summarizes the main outlines of neural network regression method.

\section{Neural Network}

A neural network is a two-stage regression or classification model, typically represented by a network diagram as in figure 4. For regression, typically $\mathrm{K}=1$ and there is only one output unit Y1 at the top [9].

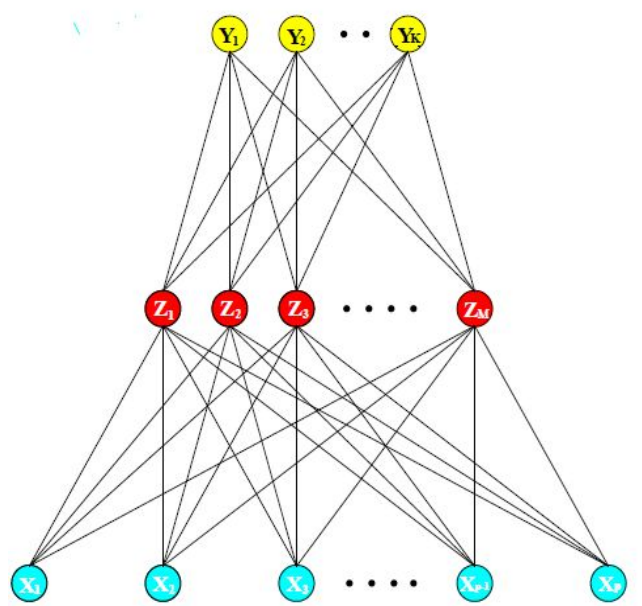

Fig.3. A Schematic of a single hidden layer, feed-forward neural network [8]

The neural network model has unknown parameters, often called weights, and we seek values for them that make the model fit the training data well. We refer the complete set of weights by $\theta$, which consists of:

$\left\{\alpha_{0 m}, \alpha_{m} ; m=1,2, . ., M\right\} \quad M(p+1)$ weights

$\left\{\beta_{0 k}, \beta_{k} ; k=1,2, . ., K\right\} K(M+1)$ weights

For regression, we use sum-of-squared errors as our measure of fit (error function):

$$
R(\theta)=\sum_{k=1}^{K} \sum_{i=1}^{N}\left(y_{i k}-f_{k}\left(x_{i}\right)\right)^{2}
$$

The generic approach to minimize $R(\theta)$ is by gradient descent, called back propagation. In the back propagation method, each hidden unit passes and receives information only to and from units that share a connection. However, backpropagation can be very slow, and for that reason it is usually not the method of choice [9].

The Levenberg-Marquardt optimization approach [10] is a nonlinear optimization method using descent gradient. This method improves the classical gradient descent algorithm. This optimization reduces significantly the algorithm convergence time (equation 5).

$$
\begin{aligned}
& w_{i+1}=w_{i}-\mu \Delta E(w) \\
& \Delta E(w)=\frac{\partial E}{\partial w}
\end{aligned}
$$


V.

HYPRID TRACKING SYSTEM

A flow diagram of the hybrid tracking system can be seen in figure 4.

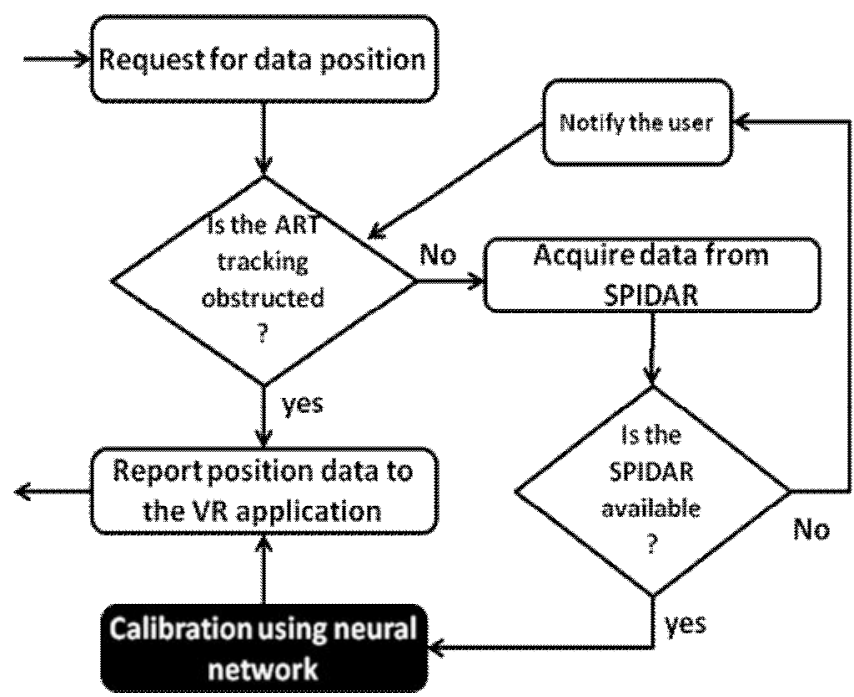

Figure 4. Flow chart of the hybrid tracking system

Once a request is being sent from the virtual reality application, the system checks whether it can provide data from the ART tracking. If this optical component loses track then the hybrid system requests position from the haptic component. In this case, the system checks the validity of data and calibrates the SPIDAR using the neural network. Switching between the two components depends on the optical tracking activity.

\section{EXPERIMENTAL RESULTS AND DISCUSSIONS}

In this section, we present the results of the neural network calibration method and we evaluate the performances of the final system. .

\section{Calibration using Neural Network}

\section{A. Neural Network implementation}

To implement a neural network we need to follow five step. First, we determine the number of input and output layers. In our work, we use one input layer which represents the position given by SPIDAR. Secondly, we scale all inputs to have mean zero and standard deviation one. This ensures all inputs are treated equally in the regularization process.

$$
\left\{\begin{array}{l}
N_{s p i}=\left(\left(P_{s p i}-\Gamma_{s p i}\right) \cdot / \sum_{s p i}\right) \\
N_{a r t}=\left(\left(P_{a r t}-\Gamma_{a r t}\right) \cdot / \sum_{a r t}\right)
\end{array}\right.
$$

Where

$$
\begin{aligned}
& \Gamma_{s p i}=\gamma_{s p i} . J \text { et } \sum_{s p i}=\sigma_{s p i} . J \\
& \Gamma_{a r t}=\gamma_{a r t} . J \text { et } \sum_{a r t}=\sigma_{a r t} . J
\end{aligned}
$$

$\gamma_{s p i}$ : Matrix of means of components of the matrix of positions given by the SPIDAR $\gamma_{\text {art }}$ : Matrix of means of components of the matrix of positions given by ART system.

$P_{s p i}:$ Positions given by the SPIDAR

$P_{a r t}:$ Positions returned by the ART system

J: Identity matrix

$N_{s p i}:$ standardized positions of the SPIDAR

$N_{a r t}$ : standardized positions of the ART system.

Thirdly, we need to estimate the number of hidden layers and units. The choice is guided by background knowledge and experimentation. With one hidden layer, network is able, with a sufficient number of units to approximate any continuous function. To avoid computation complexity, we will use one hidden layer.

The optimal number of units in the hidden layer was estimated empirically by testing many networks with different number of units. The absolute error is plotted as a function of the number of units in the hidden layer (Fig 6). The plot allows identifying the most efficient network and thus the number of units. Indeed, the number of units is equal to five.

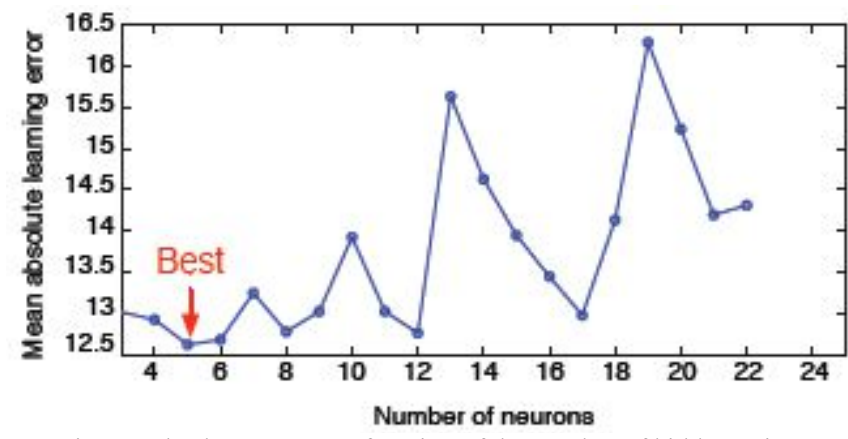

Fig. 5. Absolute error as a function of the number of hidden units

Fourthly, we use the Bipolar Sigmoid activation function to move from the input layer to the hidden layer. The second activation function is chosen to be linear.

Lastly, the learning problem that we consider in this paper is categorized as a supervised learning approach. Hence, we use a Levenberg-Marquardt algorithm, measurements of mean squared error illustrate the learning behavior.

\section{B. Neural Network performances}

We see from the graphs that the calibration via neural network was successful to reduce the magnitude of the absolute error from an almost the whole workspace (less than $30 \mathrm{~mm})$. We note that the positions returned by the neural model are very similar to those given by ART tracking system.

The magnitude of absolute error is plotted as a function of distance to the center of SPIDAR. Obviously, error is less sensitive to distance. The errors at distances higher than 400 $\mathrm{mm}$ were successfully corrected by calibration. This plot shows the efficacy of neural network. So we can easily note that the distribution of absolute position error is much more uniform after calibration. Absolutely, neural network has 
strongly reduced position error, thus increased the former accuracy.

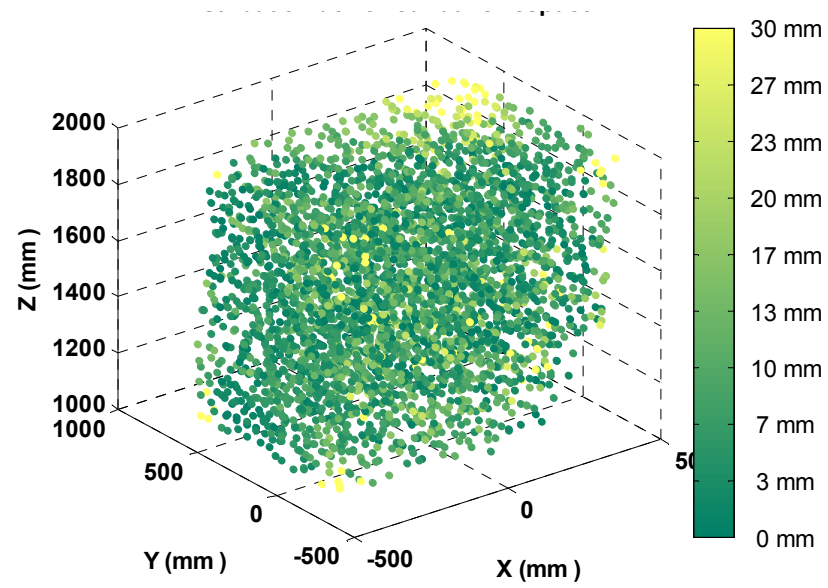

Fig. 6. Absolute Error distribution in the SPIDAR workspace after neural network calibration
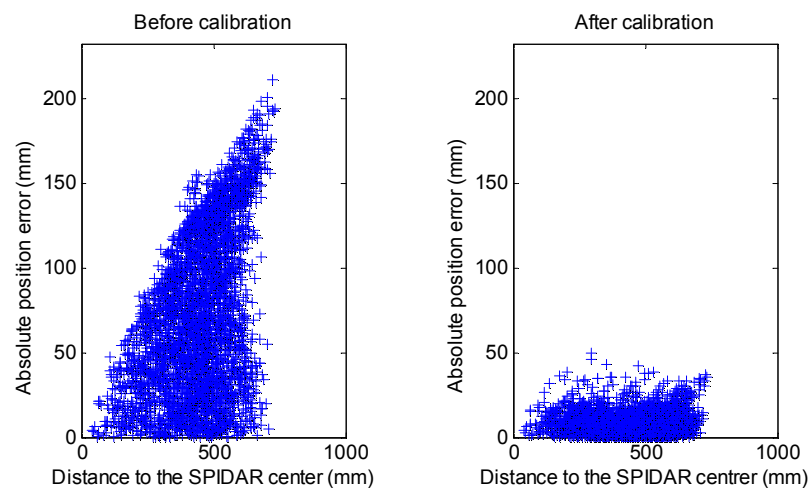

Fig. 7. Absolute position error as a function of distance to the SPIDAR center (before and after neural network based calibration)

To have better idea of the extent position error, we draw the corresponding histograms.
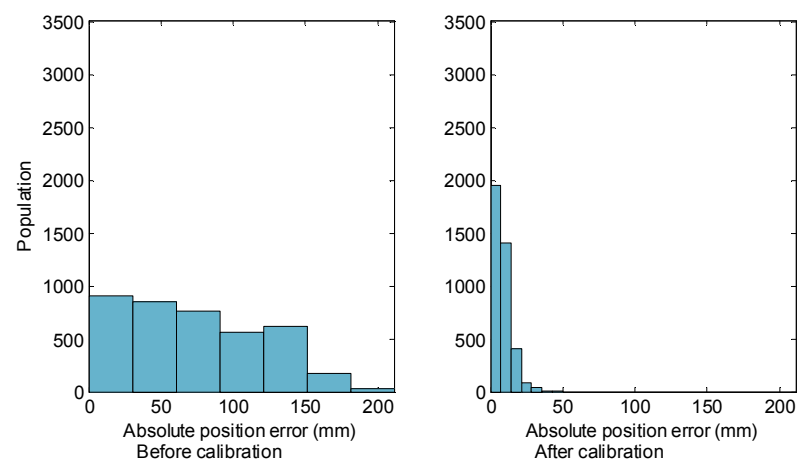

Fig. 8. Histogram of absolute position errors before and after neural network based calibration

The first histogram is spread and indicates bad position accuracy. However, the second is left shifted underlying the good accuracy of neural network results.

Neural network presents reliable standard derivation and mean error values as shown in the table 1 . In fact, before calibration, the mean absolute error reaches $72.8593 \mathrm{~mm}$ and the standard deviation is $47.0641 \mathrm{~mm}$, which means that the position given by the SPIDAR suffers from a bad accuracy and an important statistical dispersion. After calibration using neural network, the mean absolute error is below $8.1258 \mathrm{~mm}$ and the standard deviation is equal to $6.2656 \mathrm{~mm}$, meaning a higher accuracy and a lower statistical dispersion.

TABLE1. Standard deviation and the mean values

\begin{tabular}{|c|c|c|}
\multicolumn{2}{|c|}{ before and after calibration } \\
\hline Absolute Error & Raw & NN \\
\hline Mean (mm) & 72.8593 & 8.1258 \\
\hline Standard derivation $(\mathrm{mm})$ & 47.0641 & 6.2656 \\
\hline
\end{tabular}

After testing neural network with training data, we need to demonstrate its fidelity by measuring its response to measurements that it has never encountered. For this, we have created two databases to evaluate the performance of our network in generalization.

Histograms of position errors and the following tables 2 and 3 show that the neural network keeps good performance with data generalization for both databases mentioned above (database 1 and 2). The standard deviation and the mean values generated by the neural network are very good.
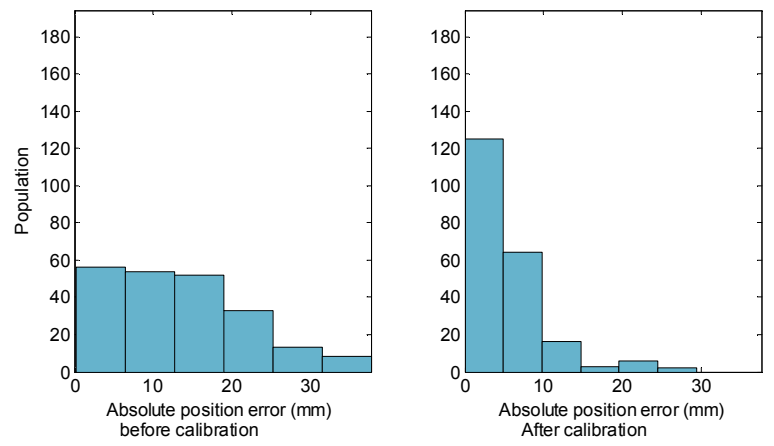

Fig. 9. Histogram of absolute position errors in generalization using the first database (database 1)

TABLE2. Standard deviation and the mean values in generalization using database 2

\begin{tabular}{|c|c|c|}
\hline Absolute Error & Raw & NN \\
\hline Mean (mm) & 13.2295 & 5.1523 \\
\hline Standard derivation (mm) & 8.4124 & 5.1177 \\
\hline
\end{tabular}



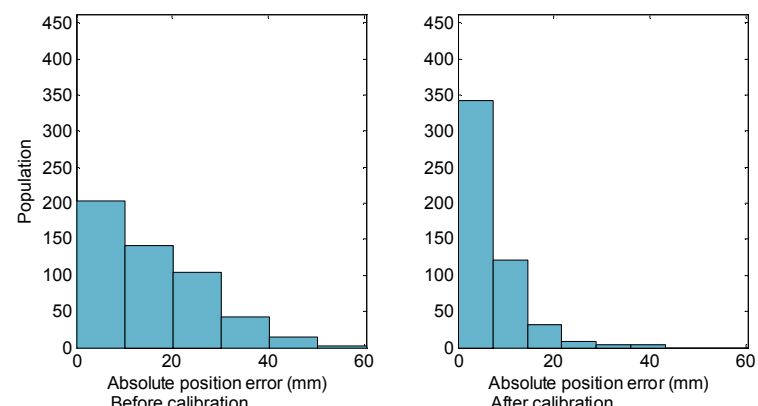

Fig. 10. Histogram of absolute position errors in generalization using the first database (database 2)

TABLE3. Standard deviation and the mean values in generalization using database 2

\begin{tabular}{|c|c|c|}
\hline Absolute Error & Raw & NN \\
\hline Mean (mm) & 15.7935 & 6.5667 \\
\hline Standard derivation (mm) & 11.6375 & 6.4320 \\
\hline
\end{tabular}

\section{Test case of Hyprid tracking system}

The haptic tracking system or SPIDAR aims to reinforce the optical tracking system. In fact, in case where the optical component loses tracking due to obstruction of the optical path or other, the system employed data provided by the SPIDAR after calibration. As shown in figure 11, this case is represented between frames 450 to 480 and 540 to 580 .

We note also that in the case where the calibration was not very effective, which can be seen in the frame 408, the optical tracking has priority.

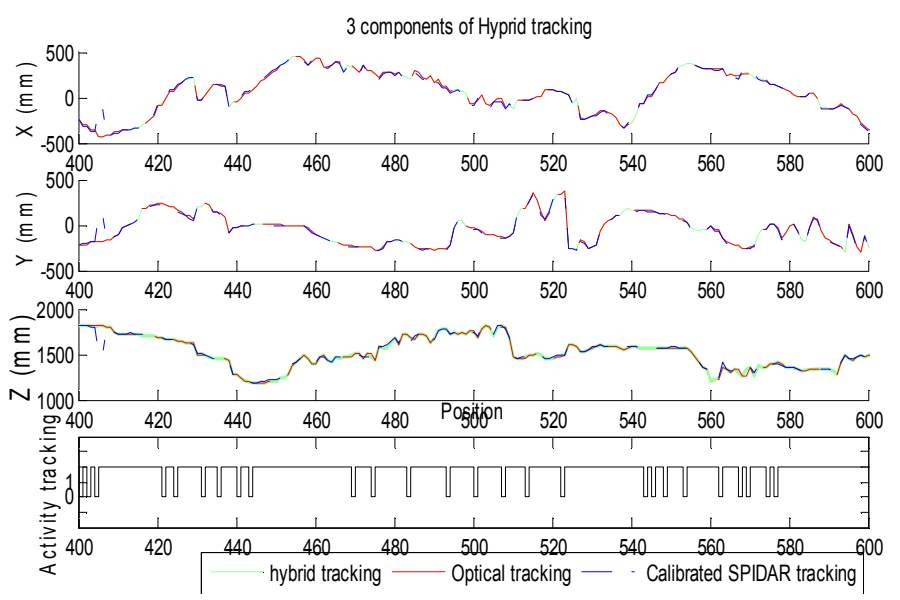

Figure 11. Example of a hybrid tracking frame

\section{CONCLUSIONS AND FURTHER WORK}

This paper proposed a method of characterization of the SPIDAR output using a virtual calibration gird. It presented also a regression method for solving the SPIDAR calibration problem and their evaluation. We have proposed also an optical-haptic tracking system that enables $3 \mathrm{D}$ interaction to be more interactive as well as more accurate and continuous.

In future work, we plan to extend the volume of workspace to improve learning by increasing the number of measurements and to test other different methods used for calibration. We intend also to take the advantages of emerging technologies like depth camera for a lower cost tracking solution in virtual and augmented reality applications.

\section{REFERENCES}

[1] Seahak Kim, Masahiro Ishii, Yasuharu Koike, and Makoto Sato, "Development of tension based haptic interface and possibility of its application to virtual reality", Proceedings of the ACM symposium on Virtual reality software and technology, pp. 199-205, 2000.

[2] Bryson S (1992) Measurement and calibration of static distortion of position data from $3 d$ trackers. Proceedings of the Special Interest Group on Graphics and Interactive Techniques,

URLhttp://citeseerx.ist.psu.edu/viewdoc/download?doi=10.1.1.140.8250\&rep $=$ rep $1 \&$ type $=$ pdf

[3] Kindratenko V, Sherman W (2005) Neural network-based calibration of electromagnetic tracking systems. Virtual Reality 9(1):70-78

[4] Barratt D, Davies A, Hughes A, Thom S (2001) Accuracy of an electromagnetic three dimensional ultrasound system for carotid artery imaging. Ultrasound in medicine \& biology 27(10):1421-1425,

URL http://linkinghub.elsevier.com/retrieve/pii/ S0301562901004471

[5] Fitzgibbon A (2003) Robust registration of $2 \mathrm{~d}$ and $3 \mathrm{~d}$ point sets. Image and Vision Computing 21(13-14):1145-1153,

URL:http://linkinghub.elsevier.com/retrieve/ pii/S0262885603001835

[6] Livingston (1997) Magnetic tracker calibration for improved augmented reality registration. Presence: Teleoperators and Virtual Environment 6(5):532-546, URL http: //eprints.kfupm.edu.sa/49599/1/49599.pdf [7] Birkfellner W, Watzinger F, Wanschitz F, Enislidis G, Truppe M, Ewers $\mathrm{R}$, Bergmann H (1998) Concepts and results in the development of a hybrid tracking system for cas. Lecture Notes in Computer Science: Medical Image Computing and Computer-Assisted Interventation 1496/1998:343,

", 2009, British Library Cataloguing-in-Publication Data.

[8] Boudoin P, Maaref H, Otmane S, Mallem M. SPIDAR Calibration based on Neural Networks versus Optical Tracking. ANNIIP'10 : Proceedings of the 6th International Workshop on Artificial Neural Networks and Intelligent Information Processing (2010).

[9] X Yan, X Gang Su, "Linear Regression Analysis: theory and computing ", 2009, British Library Cataloguing-in-Publication Data.

[10] T Hastie, R Tibshirani, J Fiedman, "The Elements of Statistical Learning: Data Mining, Inference, and Prediction", 2008, Springer Series in Statistics.

[11] Bishop, C. (2005). Neural networks for pattern recognition. OXFORD UNIVERSITY PRESS. 